\title{
Duality invariance and cosmological dynamics
}

\author{
Luis P. Chimento* and Winfried Zimdahl ${ }^{\dagger}$ \\ *Departamento de Física, Universidad de Buenos Aires 1428 Buenos Aires, Argentina \\ † Departamento de Física, Universidade Federal do Espírito Santo, CEP29060-900 Vitória, Espírito Santo, Brazil
}

(Dated: January 7, 2018)

\begin{abstract}
A duality transformation that interrelates expanding and contracting cosmological models is shown to single out a duality invariant, interacting two-component description of any irrotational, geodesic and shearfree cosmic medium with vanishing three curvature scalar. We apply this feature to a system of matter and radiation, to a mixture of dark matter and dark energy, to minimal and conformal scalar fields, and to an enlarged Chaplygin gas model of the cosmic substratum. We extend the concept of duality transformations to cosmological perturbations and demonstrate the invariance of adiabatic pressure perturbations under these transformations.
\end{abstract}

PACS numbers: 98.80.-k, 98.80 Jk, 04.40.Nr

\section{INTRODUCTION}

Different kinds of symmetries are crucial to obtain solutions of Einstein's gravitational field equations. One of the best known examples are the symmetries that underly the cosmological principle which is at the heart of most investigations in cosmology. Only the reduction of the number of degrees of freedom of the full theory by this symmetry principle makes the complicated set of nonlinear equations tractable and allows one to characterize the cosmological dynamics within Friedmann-LemaitreRobertson-Walker (FLRW) models by a few key parameters, notably the Hubble parameter which describes the expansion rate of the observable Universe. The Hubble parameter enters Friedmann's equation quadratically. This gives rise to another symmetry within a class of FLRW models itself which will be the focus of this paper. Starting point is the observation that, because of this quadratic dependence, Friedmann's equation remains invariant under a transformation $H \rightarrow-H$ for the spatially flat case. This means, it describes both expanding and contracting solutions. Such kind of duality between expanding and contracting models, except being of general theoretical interest, may be relevant, e.g., to relate post-big bang to pre-big bang background and perturbative solutions in corresponding string theoretical scenarios [1, 2, 3, 4, 5, 6, 7, 8, 9, 10]. The transformation $H \rightarrow-H$ can be seen as a consequence of a change $a \rightarrow 1 / a$ of the scale factor $a$ of the Robertson-Walker metric which is called scale factor duality [11, 12, 13, 14]. This duality becomes a true symmetry of Einstein's equations when it is supplemented by the transformations $\rho \rightarrow \rho$ of the energy density and $p \rightarrow-2 \rho-p$ of the pressure, where we assumed a perfect fluid type structure of the stress energy tensor. Under this set of transformations Friedmann's equation and the conservation equations remain invariant. Interestingly, this internal symmetry comprises both the geometrical quantity $H$ and the fluid source quantities $\rho$ and $p$. So that, when we refer to a duality transformation in the following, we have in mind the set of transformations $H \rightarrow-H, \rho \rightarrow \rho$ and $p \rightarrow-2 \rho-p$.
The aim of this paper is to discuss implications for the cosmological dynamics under the assumption of an invariance of the basic equations under duality transformations. We will uncover an underlying invariant twocomponent structure of any cosmic medium with an energy momentum tensor of the perfect fluid type. These two components have fixed equations of state and are converted into each other by a duality transformation. One of them is radiation (barotropic index $4 / 3$ ), the other one is of the phantom type and called "dual radiation" because its barotropic index is $-4 / 3$. These components represent reference fluids for the cosmic substratum. Any medium with arbitrary equation of state can be decomposed into an interacting mixture of radiation and dual radiation. The circumstance that a symmetry requirement, here the duality invariance, is accompanied by the appearance of interactions, is a feature that is well known from gauge theories. While a decomposition of the cosmic medium into radiation and dual radiation may appear artificial, its invariance properties will turn out to be useful to study the behavior of physically relevant twocomponent systems like a mixture of matter and radiation or of dark matter and dark energy. We shall also discuss realizations of the duality invariant substructure for a constant equation of state, for minimally and conformally coupled scalar fields and for an enlarged Chaplygin gas. For the case that the energy densities of both components are equal, we recover the de Sitter universe. Finally, we investigate the behavior of cosmological perturbations and show that the concept of adiabatic perturbations is duality invariant.

There is still another aspect which we would like to mention. Present observational data give strong support to an almost flat universe. This includes the possibility that the universe is indeed exactly flat $(k=0)$. Although this is an "unlikely" singular case, it deserves attention. One may speculate that a so far unknown symmetry enforces the universe to be spatially flat. If e.g., duality invariance of the kind dealt with here is a symmetry which is realized in the actual Universe, there would be no flatness problem.

The paper is organized as follows. In section $\amalg$ we 
establish our concept of duality transformations and duality invariance. In section [II we demonstrate that for any perfect fluid type cosmic medium the behavior of the relevant quantities under duality transformations naturally singles out a decomposition into a radiative fluid and a phantom fluid which we call dual radiation. We clarify the relation of this decomposition to different twocomponent descriptions of the cosmic medium. The general formalism is illustrated in section IV for a system of matter and radiation, for a mixture of dark matter and dark energy, for several scalar field configurations and for the enlarged Chaplygin gas. Section $\nabla$ is devoted to the behavior of cosmological perturbations under duality transformations. In section VI we summarize our results. Units have been chosen such that $8 \pi G=c=\hbar=1$.

\section{COSMOLOGICAL FLUID DYNAMICS}

Let us consider a cosmic medium with a perfect fluid type energy momentum tensor

$$
T^{m n}=\rho u^{m} u^{n}+p h^{m n},
$$

where $\rho$ is the energy density, $p$ is the pressure, $u^{m}$ is the four velocity normalized to $u^{m} u_{m}=-1$, and $h^{m n}=g^{m n}+u^{m} u^{n}$. Via Einstein's field equations

$$
G^{m n} \equiv R^{m n}-\frac{1}{2} g^{m n} R=T^{m n}
$$

one has

$$
G^{m n} u_{m} u_{n}=\rho, \quad \frac{1}{3} G^{m n} h_{m n}=p
$$

as well as

$$
R^{m n} u_{m} u_{n}=\frac{1}{2}(\rho+3 p), \quad R=\rho-3 p .
$$

The energy balance reads

$$
\dot{\rho}+\Theta(\rho+p)=0
$$

where $\Theta \equiv u_{; a}^{a}$ is the fluid expansion and $\dot{\rho} \equiv \rho_{, n} u^{n}$. For an irrotational, geodesic and shearfree motion the Raychaudhuri equation for $\Theta$ reduces to

$$
\dot{\Theta}+\frac{1}{3} \Theta^{2}+\frac{1}{2}(\rho+3 p)=0 .
$$

We introduce a duality transformation by a change in the sign of the expansion scalar

$$
\Theta \rightarrow \bar{\Theta}=-\Theta
$$

together with

$$
\rho \rightarrow \bar{\rho}=\rho, \quad p \rightarrow \bar{p}=-2 \rho-p .
$$

Under this transformation the energy balance 2.5 remains invariant due to $\rho+p \rightarrow-(\rho+p)$. Consequently, if the weak energy condition is satisfied in a given cosmological model, i.e. $\rho+p \geq 0$, it is violated in its dual and vice versa. In a FLRW cosmology the transformation law (2.7) implies the transformation rule $a \rightarrow \bar{a}=1 / a$ for the scale factor $a$. Accordingly, if a certain configuration (say, the unbarred one) describes a phase of contraction, the barred one describes a phase of expansion. These cosmological solutions are said to be dual to each other. In particular, there is a duality between a final contracting big crunch and a final expanding big rip. In general, the equation of state parameter will change under a duality transformation according to

$$
\bar{\gamma}=\frac{\bar{\rho}+\bar{p}}{\bar{\rho}}=-\frac{\rho+p}{\rho}=-\gamma,
$$

where $\gamma$ and $\bar{\gamma}$ are the barotropic indices of the fluid and its associated "dual fluid", respectively. The only invariant case is $p=-\rho$. This is related to the fact that the de Sitter universe is free of singularities. Using the transformation properties (2.7) for $\Theta$ and (2.8) for $\rho$ and $p$ in the Raychaudhuri equation (2.6), the latter turns out to be duality invariant if

$$
\frac{1}{3} \Theta^{2}=\rho
$$

i.e., the three curvature scalar $\mathcal{R}$ of the hypersurfaces orthogonal to $u^{a}$, in the present case given by

$$
\mathcal{R}=2\left(-\frac{1}{3} \Theta^{2}+\rho\right)
$$

has to vanish. Obviously, equation (2.11) itself is invariant under a duality transformation. The duality invariance implies the relations

$$
\bar{G}^{m n} \bar{u}_{m} \bar{u}_{n}=G^{m n} u_{m} u_{n}
$$

and

$$
\frac{1}{3} \bar{G}^{m n} \bar{h}_{m n}=-2 G^{m n} u_{m} u_{n}-\frac{1}{3} G^{m n} h_{m n}
$$


between the components of the Einstein tensor. Since energy momentum conservation is preserved under dual transformations, we have also $\bar{\nabla}_{i} \bar{G}_{k}^{i}=\nabla_{i} G_{k}^{i}$. Here we have introduced the symbol $\bar{\nabla}$ which is the usual covariant derivative with the affine connection $\Gamma_{k l}^{i}$ substituted by the corresponding duality transformed quantity $\bar{\Gamma}_{k l}^{i}$ (see below). Hence, if the Einstein equations are satisfied for a certain configuration, then a set of equations with the same form, in general, describes another potentially possible cosmological model.

In a homogeneous and isotropic background the fluid expansion in terms of the scale factor $a$ is $\Theta=3 H=$ $3 \dot{a} / a$. The equations (2.6) and (2.10) reduce to

$$
3 H^{2}=\rho, \quad \text { and } \quad-2 \dot{H}=\rho+p,
$$

respectively and they are form invariant under the duality transformation (2.7)-(2.8). In this case the former equation takes the form

$$
\bar{H}=-H \quad \rightarrow \quad \bar{a}=a^{-1} .
$$

The transformation rule of the deceleration parameter, $q \equiv-\ddot{a} /\left(a H^{2}\right)=(1+3 p / \rho) / 2$, reads

$$
\bar{q}=-q-2 .
$$

Examples are: (i) $p=\rho / 3 \Leftrightarrow q=1 \Rightarrow \bar{q}=-3$, (ii) $p=-7 \rho / 3 \Leftrightarrow q=-3 \Rightarrow \bar{q}=1$, (iii) $p=0 \Leftrightarrow q=1 / 2 \Rightarrow \bar{q}=-5 / 2$. In all these cases the sign of the deceleration parameter changes, i.e., a universe with decelerated expansion is transformed to a universe with accelerated contraction and vice versa. But for equations of state $p<-\rho / 3$ both $q$ and $\bar{q}$ are negative, e.g., (iv) $p=-2 \rho / 3 \Leftrightarrow q=-1 / 2 \Rightarrow \bar{q}=-3 / 2$. Consistent with the general setting discussed so far we have (v) $p=-\rho \Leftrightarrow q=-1 \Rightarrow \bar{q}=-1$ as the only case with $\bar{q}=q$. The cases (i) and (ii) will be of particular importance for the decomposition to be introduced in the following section. Finally, for a homogeneous and isotropic background, the non-vanishing Christoffel symbols

$$
\Gamma_{0 \nu}^{\alpha}=H \delta_{\mu}^{\alpha}, \quad \Gamma_{\mu \nu}^{0}=a^{2} H \delta_{\mu \nu}
$$

behave as

$$
\bar{\Gamma}_{0 \nu}^{\alpha}=-\Gamma_{0 \nu}^{\alpha}, \quad \bar{\Gamma}_{\mu \nu}^{0}=-\frac{1}{a^{4}} \Gamma_{\mu \nu}^{0}
$$

under a duality transformation. The transformations (2.12) and (2.13) for the Einstein tensor specify to $\bar{G}_{0}^{0}=$ $G_{0}^{0}$ and $\bar{G}_{1}^{1}=\bar{G}_{2}^{2}=\bar{G}_{3}^{3}=2 G_{0}^{0}-G_{1}^{1}$. This shows explicitly the invariance of the conservation laws under a dual transformation.

\section{TWO-COMPONENT PICTURE, GENERAL RELATIONS}

In many periods of the evolution of the Universe the cosmic medium is adequately described as a mixture of two components. The total energy and the total pressure are then split according to

$$
\rho=\rho_{A}+\rho_{B}, \quad p=p_{A}+p_{B} .
$$

A typical example is a system of matter and radiation which underlies, e.g., simple models of reheating in the early universe [15, 16]. Another example of current interest is the description of the present phase of accelerated expansion in terms of dark energy and dark matter as the two dynamically dominating components of the cosmic substratum. Now, the duality transformations (2.7) and (2.8) are independent of any underlying two- or multicomponent description. The behavior of the individual components under duality transformations remains open. There is a degeneracy with respect to a potential internal structure of the medium. What matters there are only the total energy density and the total pressure. What one would like to have is a prescription of how a given two-component structure behaves under a duality transformation, i.e., which are the counterparts $\bar{\rho}_{A}$ and $\bar{\rho}_{B}$ of $\rho_{A}$ and $\rho_{B}$, respectively. It is this question that we are interested in in the following.

A convenient starting point is the circumstance that the transformation rule of the scalar curvature (cf. (2.4) and (2.8)),

$$
R=\rho-3 p \quad \Rightarrow \quad \bar{R}=\bar{\rho}-3 \bar{p}=7 \rho+3 p,
$$

apparently singles out the duality related equations of state $p=\rho / 3$ and $p=-7 \rho / 3$, the latter being of the phantom type. Namely, for $p=\rho / 3$ the curvature scalar is zero, while the dual curvature scalar vanishes for $\bar{p}=\bar{\rho} / 3$, equivalent to $p=-7 \rho / 3$. We shall demonstrate now that these two (fixed) equations of state, which we will denote by

$$
p_{1}=\frac{\rho_{1}}{3} \quad \text { and } \quad p_{2}=-\frac{7}{3} \rho_{2}
$$

respectively, may be used as a two dimensional basis with respect to which any cosmic medium with arbitrary equation of state may formally be decomposed. The result is an interacting two component system with $\rho=\rho_{1}+\rho_{2}$ and $p=p_{1}+p_{2}$. For this singled out split it will be possible to obtain the transformation properties of the individual components 1 and 2 , i.e., to remove the mentioned degeneracy. Then, in a subsequent step we shall derive the dual counterparts $\bar{\rho}_{A}$ and $\bar{\rho}_{B}$ for any split $\rho=\rho_{A}+\rho_{B}$ of interest, in particular for a system of matter and radiation as well as a mixture of dark matter and dark energy. 
The curvature scalar and its dual in (3.2) can be combined into

$$
\frac{1}{8}(R+\bar{R})=\rho=\rho_{1}+\rho_{2}
$$

and

$$
-\frac{1}{6}(R-\bar{R})=(\rho+p)=\gamma_{1} \rho_{1}+\gamma_{2} \rho_{2}
$$

where

$$
p_{1}=\left(\gamma_{1}-1\right) \rho_{1} \quad \text { and } \quad p_{2}=\left(\gamma_{2}-1\right) \rho_{2} \text {, }
$$

with $\gamma_{1}=4 / 3$ and $\gamma_{2}=-\gamma_{1}=-4 / 3$, respectively, and

$$
\rho_{1}=\frac{\bar{R}}{8}=\frac{1}{8}(7 \rho+3 p), \quad \rho_{2}=\frac{R}{8}=\frac{1}{8}(\rho-3 p),
$$

or

$$
\rho_{1}=\frac{1}{2}\left(1+\frac{3 \gamma}{4}\right) \rho, \quad \rho_{2}=\frac{1}{2}\left(1-\frac{3 \gamma}{4}\right) \rho
$$

with $\gamma=1+p / \rho$ (cf. Eq. (2.9)). While the first component in (3.8) is a radiation component, the second (phantom type) component with a barotropic index $\gamma_{2}=-4 / 3$ will be referred to as "dual radiation". It is seen by inspection that an equation of state $p=-\rho$ is the only case in which $\bar{R}=R$. Under this condition $\dot{\bar{H}}=-\dot{H}=0$ is valid, i.e., there is a duality between de Sitter and anti-de Sitter cosmologies. Obviously, this case implies $\rho_{1}=\rho_{2}=\rho / 2$, i.e., for this configuration the energy is equally distributed on both components.

Since $\gamma$ is related to $\gamma_{1}$ and $\gamma_{2}$ via $\gamma \rho=\gamma_{1} \rho_{1}+\gamma_{2} \rho_{2}$, the balances for the components are

$$
\dot{\rho}_{1}+\Theta \gamma \rho_{1}=\frac{3}{8} \dot{\gamma} \rho
$$

and

$$
\dot{\rho}_{2}+\Theta \gamma \rho_{2}=-\frac{3}{8} \dot{\gamma} \rho
$$

or

$$
\dot{\rho}_{1}+\Theta \gamma_{1} \rho_{1}=\frac{8}{3} \Theta \frac{\rho_{1} \rho_{2}}{\rho}+\frac{3}{8} \dot{\gamma} \rho
$$

and

$$
\dot{\rho}_{2}+\Theta \gamma_{2} \rho_{2}=-\frac{8}{3} \Theta \frac{\rho_{1} \rho_{2}}{\rho}-\frac{3}{8} \dot{\gamma} \rho
$$

These relations prove our statement that under the condition of vanishing three curvature any irrotational, geodesic and shearfree cosmic substratum with effective scalar pressure $p$ and energy density $\rho$ can always be described as an interacting two-component mixture $p=$ $p_{1}+p_{2}$ and $\rho=\rho_{1}+\rho_{2}$ with $p_{1}=\left(\gamma_{1}-1\right) \rho_{1}$ and $p_{2}=\left(\gamma_{2}-1\right) \rho_{2}$ where $\gamma_{1}=4 / 3=-\gamma_{2}$. A phantom component naturally appears in this split. The specific features of the overall equation of state $p=p(\rho)$ are entirely encoded in the interaction between both components.

Rewriting Einstein's equations (2.6) and (2.10), we get

$$
\frac{\Theta^{2}}{3}=\rho_{1}+\rho_{2}
$$

and

$$
-\frac{2}{3} \dot{\Theta}=\gamma_{1}\left(\rho_{1}-\rho_{2}\right)
$$

respectively. From Eqs. (3.6) and (3.7) together with (2.8) it follows that

$$
\begin{array}{r}
\bar{\rho}_{1}=\rho_{2}, \quad \bar{\rho}_{2}=\rho_{1}, \\
\bar{p}_{1}=-\frac{p_{2}}{7}, \quad \bar{p}_{2}=-7 p_{1},
\end{array}
$$

where $\bar{p}_{1}+\bar{p}_{2}=\bar{p}$ with $\bar{p}_{1}=\left(\bar{\gamma}_{1}-1\right) \bar{\rho}_{1}$ and $\bar{p}_{2}=\left(\bar{\gamma}_{2}-1\right) \bar{\rho}_{2}$. While the individual barotropic indices

$$
\bar{\gamma}_{1}=\gamma_{1}=\frac{4}{3}, \quad \bar{\gamma}_{2}=\gamma_{2}=-\frac{4}{3}
$$

remain invariant under the dual transformation (which is different from the case investigated in [17]), the transformation behavior of $\gamma$ is given by Eq. (2.9).

The relations (3.15) and (3.16) represent the desired transformation behavior for the singled out split (3.6) (3.8), which will be applied below to physically relevant decompositions of the cosmic medium.

The two-fluid picture of radiation and dual radiation components can be seen as a symmetric representation of the Einstein equations (3.13)-(3.14) (for vanishing three curvature) under the transformations $\rho_{1} \rightarrow \rho_{2}$ and $\Theta \rightarrow-\Theta$. Local energy conservation under these circumstances takes the form

$$
\dot{\rho}=-\frac{4}{3} \Theta\left(\rho_{1}-\rho_{2}\right)
$$

and the balances (3.11) and (3.12) become

$$
\dot{\rho}_{1}+\frac{4}{3} \Theta \rho_{1}=\Gamma \rho_{2}, \quad \dot{\rho}_{2}-\frac{4}{3} \Theta \rho_{2}=-\Gamma \rho_{2}
$$

where

$$
\Gamma \rho_{2} \equiv 8 H \frac{\rho_{1} \rho_{2}}{\rho}+\frac{3}{8}\left(\frac{p}{\rho}\right)^{\cdot} \rho
$$

With the given preferred split into radiation and dual radiation, the coupling term is completely fixed by the underlying symmetry requirement. As already mentioned, 
this reminds of properties that characterize gauge theories. However, so far the relation of the expression (3.20) to physically relevant interactions remains open.

Since the expression for the energy density ratio

$$
\epsilon \equiv \frac{\rho_{2}}{\rho_{1}}=\frac{1-3 p / \rho}{7+3 p / \rho}=\frac{1-3 \gamma / 4}{1+3 \gamma / 4}
$$

is positive, the total equation of state parameter $p / \rho$ is consistently found to be in the range $-7 / 3<p / \rho<1 / 3$. Obviously, $\bar{\epsilon}=\epsilon^{-1}$ is valid. Generally, the dynamics of the ratio $\epsilon$ is governed by the equation

$$
\dot{\epsilon}=-\frac{3}{8}\left(\frac{p}{\rho}\right)^{\cdot}(1+\epsilon)^{2} .
$$

A simple example is a constant equation of state in a homogeneous and isotropic FLRW space time. For $(p / \rho)^{\circ}=0$, equivalent to $\gamma=4(1-\epsilon) / 3(1+\epsilon)=$ constant, equation (3.22) yields $\varepsilon=$ const. The energy densities scale as

$$
\rho_{1}, \rho_{2}, \rho \propto a^{-4 \frac{1-\epsilon}{1+\epsilon}} .
$$

The interaction corresponds to a decay of component 2 into component 1 , where the decay rate $\Gamma$, according to Eq. (3.20), is given by

$$
\Gamma=8 H \frac{\rho_{1}}{\rho}=8 H \frac{1}{1+\epsilon},
$$

i.e., the rate $\Gamma$ is proportional to the Hubble rate. Although the component 2 decays, the ratio $\epsilon=\rho_{2} / \rho_{1}$ remains constant. This is due to the circumstance that $\gamma_{2}$ is negative, i.e., without interaction $\rho_{2}$ would grow with the expansion (for $H>0$ ). Growth and decay are balanced such that the ratio of the energy densities is unaltered.

Interesting subcases are: (i) radiation, $\gamma=4 / 3$, equivalent to $\rho_{1}=\rho$ and $\epsilon=0$, (ii) dust $\gamma=1$, where $\rho_{1}=7 \rho / 8$ and $\epsilon=1 / 7$, (iii) vacuum energy, $\gamma=0$, characterized by $\rho_{1}=\rho / 2$ and $\epsilon=1$, (iv) dual radiation, $\gamma=-4 / 3$, for which $\rho_{1}=0$ and $\epsilon \rightarrow \infty$.

Now, there will be hardly an epoch in which the decomposition into radiation and dual radiation with a specific interaction between both components is a physically adequate description of the cosmic medium, although it is formally always valid. However, the duality invariance of this split can be used to characterize the duality behavior of other, more realistic two-component descriptions, which by themselves are not duality invariant.

Let us consider to this purpose a different decomposition of the cosmic medium according to (3.1) with

$$
p_{A}=\left(\gamma_{A}-1\right) \rho_{A}, \quad p_{B}=\left(\gamma_{B}-1\right) \rho_{B} .
$$

The relations between the duality invariant split (3.7) and the arbitrary decomposition (3.1) are

$$
\rho_{1}=\frac{1}{8}\left[\left(4+3 \gamma_{A}\right) \rho_{A}+\left(4+3 \gamma_{B}\right) \rho_{B}\right]
$$

and

$$
\rho_{2}=\frac{1}{8}\left[\left(4-3 \gamma_{A}\right) \rho_{A}+\left(4-3 \gamma_{B}\right) \rho_{B}\right]
$$

or

$$
\rho_{A}=\left[\frac{\gamma_{B}-\frac{4}{3}}{\gamma_{B}-\gamma_{A}} \rho_{1}+\frac{\gamma_{B}+\frac{4}{3}}{\gamma_{B}-\gamma_{A}} \rho_{2}\right]
$$

and

$$
\rho_{B}=\left[\frac{\gamma_{A}-\frac{4}{3}}{\gamma_{A}-\gamma_{B}} \rho_{1}+\frac{\gamma_{A}+\frac{4}{3}}{\gamma_{A}-\gamma_{B}} \rho_{2}\right] .
$$

Of course, this satisfies

$$
\rho_{1}+\rho_{2}=\rho_{A}+\rho_{B},
$$

while the difference $\rho_{1}-\rho_{2}$ which appears in (3.18), transforms into

$$
\rho_{1}-\rho_{2}=\frac{3}{4}\left[\gamma_{A} \rho_{A}+\gamma_{B} \rho_{B}\right] .
$$

The transformation properties (3.15) and (3.16) of $\rho_{1}, \rho_{2}$ and $p_{1}, p_{2}$, respectively, can now be used to obtain the corresponding behavior of $\rho_{A}, \rho_{B}, p_{A}$ and $p_{B}$. In the following we discuss several applications of this scheme.

\section{TWO-COMPONENT PICTURE, APPLICATIONS}

\section{A. Matter and radiation}

As a first application we consider a mixture of matter (subscript $m$ ) and radiation (subscript $r$ ),

$$
p_{m}=0, \quad p_{r}=\frac{\rho_{r}}{3} .
$$

With

$$
\gamma_{A} \equiv \gamma_{m}=1, \quad \gamma_{B} \equiv \gamma_{r}=\frac{4}{3}
$$

we obtain from (3.28) and (3.29),

$$
\rho_{m}=8 \rho_{2}, \quad \rho_{r}=\rho_{1}-7 \rho_{2},
$$

or, from (3.26) and (3.27),

$$
\rho_{1}=\frac{7}{8} \rho_{m}+\rho_{r}, \quad \rho_{2}=\frac{\rho_{m}}{8} .
$$

It follows that

$$
\rho_{1}-\rho_{2}=\frac{3}{4} \rho_{m}+\rho_{r} .
$$

The behavior under duality transformations is

$$
\bar{\rho}_{m}=7 \rho_{m}+8 \rho_{r}, \quad \bar{\rho}_{r}=-6 \rho_{m}-7 \rho_{r},
$$


guaranteeing $\bar{\rho}_{m}+\bar{\rho}_{r}=\rho_{m}+\rho_{r}$.

Equation (3.18) can be written as

$$
\dot{\rho}_{m}+3 H \rho_{m}+\dot{\rho}_{r}+4 H \rho_{r}=0,
$$

which is equivalent to

$$
\begin{gathered}
\dot{\rho}_{m}+3 H \rho_{m}=-Q, \\
\dot{\rho}_{r}+4 H \rho_{r}=Q,
\end{gathered}
$$

where

$$
Q=(\Gamma-7 H) \rho_{m} .
$$

The set of equations (4.8) - 4.10) describes matter in interaction with radiation. While $\Gamma$ characterizes the interaction between radiation and dual radiation and may be considered a fictitious interaction rate, the quantity $Q$ is supposed to describe a real physical interaction, e.g., the decay of a scalar field into radiation in simplified reheating models of inflationary scenarios 15, , 16]. However, only the knowledge of the duality transformation behavior $\overline{\Gamma \rho}_{2} \rightarrow-\Gamma \rho_{2}$ of (3.20) allows us to obtain the dual counterpart $\bar{Q}$ of $Q$. The combinations

$$
Q+\bar{Q}=56 H\left(\rho_{1}-\rho_{2}\right)
$$

and

$$
Q-\bar{Q}=16 \Gamma \rho_{2}-56 H\left(\rho_{1}+\rho_{2}\right)
$$

are obviously duality invariant. The quantity $Q$ itself, however, changes under a duality transformation. From (4.10) we find that the non-interacting case $Q=0$ corresponds to $\Gamma=7 H$. Its dual $\bar{Q}$, on the other hand, is different from zero. Consequently, the dual of a noninteracting mixture of matter and radiation is interacting. From

$$
\bar{p}_{m}+\bar{p}_{r}=\frac{1}{3} \bar{\rho}_{r}=-2 \rho_{m}-\frac{7}{3} \rho_{r}
$$

and

$$
\bar{p}_{m}-\bar{p}_{r}=-\frac{1}{3} \bar{\rho}_{r}=-\left(\bar{p}_{m}+\bar{p}_{r}\right)
$$

we obtain the corresponding transformed equations of state

$$
\bar{p}_{m}=0, \quad \bar{p}_{r}=\frac{1}{3} \bar{\rho}_{r},
$$

i.e., the individual equations of state preserve their form.

\section{B. Dark matter and dark energy}

Another interesting special case is a mixture of matter, $\gamma_{M} \equiv \gamma_{A}=1$ and dark energy $\gamma_{X} \equiv \gamma_{B}=0$. Then (cf. (3.26) - (3.29)

$$
\rho_{1}=\frac{1}{2} \rho_{X}+\frac{7}{8} \rho_{M}
$$

and

$$
\rho_{2}=\frac{1}{2} \rho_{X}+\frac{1}{8} \rho_{M}
$$

or

$$
\rho_{M}=\frac{4}{3}\left(\rho_{1}-\rho_{2}\right)
$$

and

$$
\rho_{X}=-\frac{1}{3} \rho_{1}+\frac{7}{3} \rho_{2}
$$

With these transformations the balance equation (3.18) takes the form

$$
\dot{\rho}_{M}+3 H \rho_{M}=-\dot{\rho}_{X} .
$$

This represents a model in which matter and dark energy are in general interacting with each other:

$$
\dot{\rho}_{M}+3 H \rho_{M}=Q, \quad \dot{\rho}_{X}=-Q,
$$

with

$$
Q=-\frac{4}{3} H\left(4 \rho_{X}+\frac{7}{4} \rho_{M}\right)+\frac{4}{3} \Gamma\left(\rho_{X}+\frac{1}{4} \rho_{M}\right) .
$$

Under duality transformations the variables of this model behave as

$$
\bar{\rho}_{M}=-\rho_{M}, \quad \bar{\rho}_{X}=\rho_{X}+2 \rho_{M} .
$$

The combinations

$$
Q+\bar{Q}=8 H\left(\rho_{1}-\rho_{2}\right)
$$

and

$$
\left.Q-\bar{Q}=\frac{16}{3}\left[\Gamma \rho_{2}-2 H\left(\rho_{1}+\rho_{2}\right)\right)\right]
$$

are duality invariant.

The $\Lambda \mathrm{CDM}$ model is realized for $Q=0$. Its dual counterpart is an interacting model with

$$
\bar{Q}=8 H\left(\rho_{1}-\rho_{2}\right) \quad \Rightarrow \quad \Gamma=\frac{H}{2} \frac{\rho_{1}+7 \rho_{2}}{\rho_{2}} .
$$

Any case $Q \neq 0$ corresponds to an interaction between dark matter and dark energy. Models of this type have attracted considerable attention in the literature, in particular with respect to the coincidence problem [18, 19]

Similarly to the previous example, the transformed equations of state are obtained via

$$
\bar{p}_{M}+\bar{p}_{X}=-\left(\bar{p}_{M}+\bar{p}_{X}\right) .
$$

The result is

$$
\bar{p}_{M}=0, \quad \bar{p}_{X}=-\bar{\rho}_{X} .
$$

Again, the equations of state are form-invariant while the interaction rate in the original model differs from the corresponding rate in the transformed model. 


\section{Systems with one constant component}

The balance equations (3.18) - 3.20) simplify if one of the components remains constant. For $\dot{\rho}_{2}=0$, e.g., it follows from (3.19) that $\Gamma=4 H$. This corresponds to the case $\gamma_{A}=4 / 3$ and $\gamma_{B}=0$ in 3.31). Then $\rho_{1}-\rho_{2}=$ $\rho_{A} \equiv N \propto a^{-4}$ and $\rho_{B} \equiv \Lambda=$ const. Similarly, for $\dot{\rho}_{1}=0$ one has $\Gamma=4 H \rho_{1} / \rho_{2}$ and $\rho_{2}-\rho_{1} \equiv F \propto a^{4}$. For these configurations the radiation and dual radiation components can be written as

$$
\rho_{1}=N+\frac{\Lambda}{2}, \quad \rho_{2}=\frac{\Lambda}{2},
$$

or

$$
\rho_{1}=\frac{\Lambda}{2}, \quad \rho_{2}=F+\frac{\Lambda}{2},
$$

where $\rho=N+\Lambda$ or $\rho=F+\Lambda$ and $\Lambda$ is constant for the given dynamics. However, $\Lambda$ changes under a duality transformation. The quantities $N(F)$ and $\Lambda$ transform as

$$
\bar{N}(\bar{F})=-N(F), \quad \bar{\Lambda}=2 N(F)+\Lambda .
$$

Obviously, Eq. (4.29) describes a universe filled with radiation and a cosmological constant, while Eq. (4.30) is an analogous configuration with radiation replaced by dual radiation.

Because of the mentioned dependence on the scale factor $N$ and $F$ can be written as

$$
N=\frac{N_{0}}{a^{4}}, \quad F=F_{0} a^{4},
$$

where $N_{0}$ and $F_{0}$ are integration constants. In terms of $N$ (or $F$ ) and $\Lambda$ the Friedmann equation (3.13) becomes

$$
3 H^{2}=\frac{N_{0}}{a^{4}}+\Lambda, \quad \text { or } \quad 3 H^{2}=F_{0} a^{4}+\Lambda .
$$

Integration of equations (4.33) provides us with the scale factors

$$
\begin{array}{ll}
a_{N}^{ \pm}=\left[ \pm \sqrt{\frac{N_{0}}{\Lambda}} \sinh \sqrt{\frac{4 \Lambda}{3}} t\right]^{1 / 2} \quad N_{0}>0, \\
a_{N}=\left[\sqrt{\frac{-N_{0}}{\Lambda}} \cosh \sqrt{\frac{4 \Lambda}{3}} t\right]^{1 / 2} \quad N_{0}<0,
\end{array}
$$

in the radiation case and

$$
a_{F}^{ \pm}=\frac{1}{a_{N}^{ \pm}\left(N_{0}=F_{0}\right)}, \quad a_{F}=\frac{1}{a_{N}\left(N_{0}=F_{0}\right)},
$$

for the dual radiation case. The solution $a_{F}^{-}$, defined in the region $t<0$, describes a big rip at $t=0$. On the other hand, the super-accelerated solution $a_{N}$ has an infinite time span and a final de Sitter stage.

\section{The minimal scalar field}

In this part we derive an explicit scalar field dynamics which is a realization of the structure (4.29) - 4.30) with the results (4.34) - 4.36). Starting point are the expressions

$$
\rho_{\phi}=\frac{1}{2} \dot{\phi}^{2}+V(\phi) \quad \text { and } \quad p_{\phi}=\frac{1}{2} \dot{\phi}^{2}-V(\phi)
$$

for the energy density $\rho_{\phi}$ and the pressure $p_{\phi}$, respectively, of a scalar field. The decomposition into radiation and dual radiation according to Eq. (3.7) then yields

$$
\rho_{1}=\frac{1}{8}\left[5 \dot{\phi}^{2}+4 V\right], \quad \rho_{2}=\frac{1}{8}\left[-\dot{\phi}^{2}+4 V\right],
$$

where the first component represents some kind of quintessential scalar field while the second component is associated with a phantom scalar field. Independent of any interaction between the two fluid components, the energy-momentum tensor conservation of the system as a whole is equivalent to the Klein-Gordon equation. Applying the transformation rules (2.8) to $\rho_{\phi}$ and $p_{\phi}$ of (4.37), we obtain the transformation properties for the kinetic energy and for the potential of the scalar field:

$$
\dot{\bar{\phi}}^{2}=-\dot{\phi}^{2}, \quad \bar{V}=V+\dot{\phi}^{2} .
$$

To see how the structure 4.29 - 4.30 applies to the scalar field dynamics, it is convenient to choose the potentials

$$
V_{s}=\frac{1}{4} \dot{\phi}^{2}+\Lambda \quad \text { and } \quad V_{p h}=-\frac{5}{4} \dot{\phi}^{2}+\Lambda,
$$

in (4.38). As will become clear in a moment, $V_{s}$ and $V_{p h}$ are associated with "conventional" scalar (subscript s) and phantom (subscript ph) fields, respectively. Namely, with (4.40) the components $\rho_{1}$ and $\rho_{2}$ in (4.38) take the form

$$
\rho_{1 s}=\frac{3}{4} \dot{\phi}^{2}+\frac{\Lambda}{2} \quad \text { and } \quad \rho_{2 s}=\frac{\Lambda}{2},
$$

respectively, or

$$
\rho_{1 p h}=\frac{\Lambda}{2} \quad \text { and } \quad \rho_{2 p h}=-\frac{3}{4} \dot{\phi}^{2}+\frac{\Lambda}{2} .
$$

These components have indeed the structure of 4.29) and (4.30) with $N=F_{s}=3 \dot{\phi}^{2} / 4=-F_{p h}=-F$. Therefore, the corresponding scale factors are given by (4.34)(4.36). Inserting the non-phantom and phantom fluids (4.41) - (4.42) into the balance equations (3.11)-(3.12) and integrating, we obtain the first integrals

$$
\dot{\phi}_{s}=\sqrt{\frac{4 N_{0}}{3}} a^{-2} \text { and } \quad \dot{\phi}_{p h}=\sqrt{-\frac{4 F_{0}}{3}} a^{2}
$$


for the scalar (non-phantom) and phantom field cases, respectively. The explicit scalar field dynamics is then derived by using Eqs. (4.34)-(4.36) in (4.43). For the potentials (4.40) we find

$$
V_{s}=\Lambda\left[\cosh ^{2} \phi-\frac{2}{3} \sinh ^{2} \phi\right]
$$

with

$$
\phi=\ln \tanh \sqrt{\frac{\Lambda}{3}} t, \quad N_{0}>0,
$$

and

$$
V_{s}=\Lambda\left[\cos ^{2} i \phi+\frac{2}{3} \sin ^{2} i \phi\right]
$$

with

$$
\phi=-2 i \arctan e^{\sqrt{4 \Lambda / 3} t}, \quad N_{0}<0,
$$

or,

$$
V_{p h}=\Lambda\left[\cosh ^{2} i \phi+\frac{2}{3} \sinh ^{2} i \phi\right]
$$

with

$$
\phi=-i \ln \tanh \sqrt{\frac{\Lambda}{3}} t, \quad F_{0}>0
$$

and

$$
V_{p h}=\Lambda\left[\cos ^{2} \phi-\frac{2}{3} \sin ^{2} \phi\right]
$$

with

$$
\phi=2 \arctan e^{\sqrt{4 \Lambda / 3} t}, \quad N_{0}<0
$$

We mention again, that the duality related components $\rho_{1}$ and $\rho_{2}$ in Eq. (4.38) describe a quintessence-type scalar field and a phantom field, respectively. This is quite similar to features which are characteristic for "quintom" models of the cosmic substratum 20]. A mixture of this type has attracted considerable interest recently since it may describe the crossing of the so called phantom divide which seems to be favored by a number of observational data [21, 22].

\section{The conformal scalar field}

An interesting particular case is obtained for a vanishing $N$, corresponding to the one-component limit $\rho_{1}-\rho_{2}=\rho_{A}=0$. Namely, for $N=0$ the constant $\Lambda$ preserves its form, i.e., $\bar{\Lambda}=\Lambda$ (cf. Eq. (4.31)). We provide a representation of this case by a conformal scalar field $\psi$ with the potential $V(\psi)=\lambda \psi^{4}+\Lambda$ with $\Lambda>0$.
This potential has received much attention in the literature in connection with the early inflationary epoch [23]. This simplified model leads to a final accelerated expansion phase retaining the essentials of minimally coupled approaches. The energy density and the pressure of the conformal scalar field are

$$
\begin{aligned}
\rho_{\psi} & =\frac{1}{2}(\dot{\psi}+H \psi)^{2}+\lambda \psi^{4}+\Lambda, \\
p_{\psi} & =\frac{1}{6}(\dot{\psi}+H \psi)^{2}+\frac{\lambda}{3} \psi^{4}-\Lambda .
\end{aligned}
$$

The radiation dual radiation decomposition (3.7) of this conformal scalar field leads to

$$
\rho_{1}=\frac{1}{2}\left[(\dot{\psi}+H \psi)^{2}+2 \lambda \psi^{4}\right]+\frac{\Lambda}{2}, \quad \rho_{2}=\frac{1}{2} \Lambda .
$$

Comparing the first equations in (4.29) and (4.32) with (4.54), we get

$$
\frac{1}{2}(\dot{\psi}+H \psi)^{2}+\lambda \psi^{4}=\frac{N_{0}}{a^{4}},
$$

and its corresponding dual (barred) counterpart. Applying the transformation rule (3.15) to the components (4.54), we obtain

$$
\begin{aligned}
\frac{1}{2}(\dot{\bar{\psi}}+\bar{H} \bar{\psi})^{2}+\bar{\lambda} \bar{\psi}^{4} & =-\frac{1}{2}(\dot{\psi}+H \psi)^{2}-\lambda \psi^{4}, \\
\bar{\Lambda} & =\Lambda+(\dot{\psi}+H \psi)^{2}+2 \lambda \psi^{4}
\end{aligned}
$$

Now we compare Eqs. (4.55), its dual (barred), and (4.56). It follows that $\bar{N}_{0} / \bar{a}^{4}=-N_{0} / a^{4}=\bar{N}_{0} a^{4}$. This has a solution $\bar{N}_{0}=N_{0}=0$, for which $\bar{\Lambda}=\Lambda$ (cf. Eqs. (4.31) and (4.32). Consequently, $\bar{N}=-N=0$, which is just a realization of the special situation mentioned above. In this case the Friedmann equation simplifies to $3 H^{2}=\Lambda$, with the de Sitter solution $a=e^{H_{0} t}$, where $H_{0}= \pm \sqrt{\Lambda / 3}$.

Integrating Eq. (4.56) we obtain the conformal field

$$
\psi=\frac{H_{0}}{\sqrt{-2 \lambda}\left[1-e^{H_{0} t}\right]}, \quad \lambda<0,
$$

and its transformation rule

$$
\bar{\psi}=\sqrt{\frac{\lambda}{\bar{\lambda}}}\left[\psi-\frac{H_{0}}{\sqrt{-2 \lambda}}\right] .
$$

So, the duality invariance of the de Sitter solution induces a linear transformation group acting on $\psi$. In the particular case of $\bar{\lambda}=\lambda$, i.e., for an invariance of the field strength, the radiation - dual radiation decomposition produces a simple translation of the conformal field.

This completes our discussion of the internal structure generated by our duality invariant two-component description when applied to a (minimal or conformal) scalar 
field dynamics. It is expedient to notice that the underlying decompositions (4.29) and (4.30) are relations in which the energy densities of the components, $\rho_{1}$ and $\rho_{2}$, depend linearly on the total energy density $\rho$. In the following subsection we shall perform a fluid dynamical analysis for an enlarged Chaplygin gas, for which the corresponding decomposition is non-linear.

\section{Enlarged Chaplygin gas}

So far we have emphasized that a given cosmological model can be decomposed with respect to a duality invariant two-component basis. Here we focus on the inverse procedure. Assuming a fairly general non-linear structure for $\rho_{1}$ and $\rho_{2}$ as a starting point, we shall construct an effective equation of state for the cosmic medium as a whole. More specifically, we shall derive in this way a set of cosmologies (first considered in [24]) which were called enlarged Chaplygin cosmologies in 25]. This class includes as particular cases generalized [26], extended and modified Chaplygin cosmologies 27] along with their duals. This larger class of Chaplygin cosmologies, which can be induced using duality transformations [25], represents a further generalization of the extended and modified Chaplygin gas scenarios, because it includes both super-accelerated and contracting cosmologies.

We start by introducing general radiation and dual radiation components

$$
\rho_{1}=b \rho+J(\rho) \quad \text { and } \quad \rho_{2}=(1-b) \rho-J(\rho),
$$

respectively, where $b$ is a constant and $J(\rho)$ is an arbitrary, in general non-linear, function of the total energy density. Of course, $\rho=\rho_{1}+\rho_{2}$ is valid. The transformation rules (3.15) imply

$$
J+\bar{J}+(b+\bar{b}) \rho=\rho .
$$

For this configuration the overall barotropic index $\gamma=$ $4\left(\rho_{1}-\rho_{2}\right) / 3 \rho$ is

$$
\gamma=\frac{4}{3}(2 b-1)+\frac{8 J}{3 \rho},
$$

while the equation of state of the total fluid is given by

$$
\frac{p}{\rho}=\frac{1}{3}(8 b-7)+\frac{8 J}{3 \rho} .
$$

Now we choose

$$
b=\frac{1}{2}+\frac{3 \gamma_{0}}{8} \quad \text { and } \quad J=-\frac{3 \gamma_{0} A}{8 \rho^{\alpha}}
$$

with constants $\gamma_{0}, A$ and $\alpha$. By this procedure we are led to the equation of state for the enlarged Chaplygin gas,

$$
\frac{p}{\rho}=\gamma_{0}-1-\gamma_{0} \frac{A}{\rho^{\alpha+1}} .
$$

It results in an energy density

$$
\rho=\left[A+\frac{B}{a^{3 \gamma_{0}(1+\alpha)}}\right]^{1 / 1+\alpha},
$$

with an arbitrary integration constant $B$. It is convenient to introduce the quantity $y \equiv A a^{3 \gamma_{0}(1+\alpha)} / B$. The duality transformations (2.8) require that $\overline{\gamma_{0}}=-\gamma_{0}$ and $\bar{a}=1 / a$, which guarantees the invariance of $y$. This behavior is also consistent with the transformation rule (2.9) of the overall barotropic index $\gamma=\gamma_{0} /(1+y)$. For the time dependence of the energy density ratio $\epsilon$ we find

$$
\dot{\epsilon}=\frac{9}{8} H(\alpha+1) \gamma_{0}^{2}(1+\epsilon)^{2} \frac{y}{(1+y)^{2}} .
$$

For any $H>0$ and $y>0$ the ratio $\epsilon$, given by (3.21), increases. For large values of the scale factor, corresponding to large $y$ - values, the quantity $\gamma$ tends to zero, i.e., $p \rightarrow-\rho, \epsilon \rightarrow \epsilon_{\mathrm{f}}=1$ and the scale factor tends to the stable (see below) de Sitter solution.

For small values of the scale factor, equivalent to small values of $y$, i.e., at an early cosmological epoch, we have $\gamma \rightarrow \gamma_{0}$ and $\epsilon$ tends to the constant initial value $\epsilon_{i}=\epsilon\left(\gamma_{0}\right)$. Remarkably, all these limits are independent of $\alpha$. There is an evolution of $\epsilon$ from $\epsilon_{\mathrm{i}}<1$ to $\epsilon=1$. For the latter value, equivalent to $p=-\rho$, we recover the expected result that the energy densities of radiation and dual radiation coincide. The evolution of the density ratio reminds of a similar feature in interacting cosmological models in which a suitable interaction is used to address the coincidence problem [28]. The decay rate (cf. Eq. (3.20) ) by which energy is transferred from component 2 to component one is

$$
\Gamma=4 H\left(1+\frac{3}{4} \gamma\right)-\frac{9}{4}(\alpha+1) H \frac{\gamma^{2} y}{1-3 \gamma / 4} .
$$

It changes between $\Gamma_{\text {early }}=4 H\left(1+3 \gamma_{0} / 4\right)$ for $y \ll 1$ at small values of the scale factor to $\Gamma_{\text {late }}=4 H$ for $y \gg 1$ in the late time limit with $\epsilon=\epsilon_{\mathrm{f}}=1$. These limits of the decay rate coincide with those obtained from (3.20). So, the enlarged Chaplygin gas interpolates between a scaling era and a de Sitter scenario.

To investigate the stability properties of the stationary solution we consider $\epsilon=\epsilon_{0}+\delta$ with $\dot{\epsilon}_{0}=0$ and assume that $\delta$ is an arbitrary function. Inserting this expression for $\epsilon$ in Eq. (3.21) we find

$$
\dot{\delta}=-\frac{24}{\left(4+3 \gamma_{0}\right)^{2}} \dot{\gamma}
$$

where

$$
\dot{\gamma}=-3 H(1+\alpha) \gamma_{0}^{2} \frac{y}{(1+y)^{2}} .
$$

There are two cases: 
a) If $\delta>0$ with $\dot{\delta}<0$ and $\dot{\gamma}>0$, then $(1+\alpha) y<0$ and the constant solution $\epsilon_{0}$ is stable.

b) If $\delta<0$ with $\dot{\delta}>0$ and $\dot{\gamma}<0$, then $(1+\alpha) y>0$ and the constant solution $\epsilon_{0}$ is stable as well.

Case b) covers the physically most interesting configuration $A>0, B>0, \alpha>0$. In particular, the stability property holds for the asymptotic solution $\epsilon_{0}=\epsilon_{f}=1$.

Finishing this part, we mention that in the literature there exist other types of decompositions of Chaplygin gases which describe two-component mixtures of dark matter and dark energy [29, 30]. These different splits (and their behavior under duality transformations) may be recovered from the present split into radiation and dual radiation in a similar way in which, e.g, the $\Lambda \mathrm{CDM}$ model was obtained in subsection IVB.

\section{PERTURBATION DYNAMICS AND DUALITY}

So far we have studied implications of a duality motivated split of the cosmological dynamics under the conditions of spatial homogeneity and anisotropy. However, the formalism outlined in sections [I] and [II is more general. It allows us to split the entire dynamics into a homogeneous and isotropic background part, described in section [IV, and first-order perturbations about this background. All the quantities $\rho, p, \Theta$ are assumed to decompose according to

$$
\rho=\rho_{(b)}+\hat{\rho}, \quad p=p_{(b)}+\hat{p}, \quad \Theta=\Theta_{(b)}+\hat{\Theta}, \ldots
$$

where the subscript $(b)$ stands for background. We recall that the duality transformations (2.7), (2.8), (3.15) (3.16), (3.17) were supposed to hold for the total quantities $\rho, p, \Theta$, etc. On the other hand, all the previous applications relied on the fact that these relations are separately valid in the background, i.e.,

$$
\bar{\rho}_{(b)}=\rho_{(b)}, \quad \bar{p}_{(b)}=-2 \rho_{(b)}-p_{(b)}, \quad \bar{\Theta}_{(b)}=-\Theta_{(b)}, \ldots .
$$

Perturbations of the duality transformed quantities are introduced by

$$
\bar{\rho}=\bar{\rho}_{(b)}+\hat{\bar{\rho}}, \quad \bar{p}=\bar{p}_{(b)}+\hat{\bar{p}}, \quad \bar{\Theta}=\bar{\Theta}_{(b)}+\hat{\bar{\Theta}}, \ldots .
$$

Taking here (5.2) into account leads to the transformation behavior

$$
\hat{\bar{\rho}}=\hat{\rho}, \quad \hat{\bar{p}}=-2 \hat{\rho}-\hat{p}, \quad \hat{\bar{\Theta}}=-\hat{\Theta} \ldots .
$$

of the perturbations. Also, the relations

$$
\hat{\bar{\rho}}_{1}=\hat{\rho}_{2}, \quad \hat{\bar{\rho}}_{2}=\hat{\rho}_{1}, \quad \hat{\bar{p}}_{1}=-\frac{1}{7} \hat{p}_{2}, \quad \hat{\bar{p}}_{2}=-7 \hat{p}_{1},
$$

are valid.

\section{A. Matter perturbations}

To study perturbations about the homogeneous and isotropic background we introduce the following quantities (cf. [30]). We define the total fractional energy density perturbation

$$
D \equiv \frac{\hat{\rho}}{\rho+p}=-3 H \frac{\hat{\rho}}{\dot{\rho}},
$$

and the energy density perturbations for the components $(A=1,2)$

$$
D_{A} \equiv-3 H \frac{\hat{\rho}_{A}}{\dot{\rho}_{A}}
$$

(The subscript (b) will be omitted from now on.) Under a duality transformation these quantities behave as

$$
\bar{D}=-D, \quad \bar{D}_{1}=-D_{2}, \quad \bar{D}_{2}=-D_{1} .
$$

The fractional energy density perturbations of component 1 transform into corresponding perturbations of component 2 with a reversed sign and vice versa. The total energy density perturbations just change their sign. The pressure perturbations are

$$
P \equiv \frac{\hat{p}}{\rho+p}=-3 H \frac{\hat{p}}{\dot{\rho}}=-3 H \frac{\dot{p}}{\dot{\rho}} \frac{\hat{p}}{\dot{p}},
$$

and

$$
P_{A} \equiv-3 H \frac{\hat{p}_{A}}{\dot{\rho}_{A}}=-3 H \frac{\dot{p}_{A}}{\dot{\rho}_{A}} \frac{\hat{p}_{A}}{\dot{p}_{A}}
$$

They transform as

$$
\bar{P}=P+2 D, \quad \bar{P}_{1}=\frac{1}{7} P_{2}, \quad \bar{P}_{2}=7 P_{1} .
$$

One also realizes that

$$
D=\frac{\dot{\rho}_{1}}{\dot{\rho}} D_{1}+\frac{\dot{\rho}_{2}}{\dot{\rho}} D_{2}
$$

and

$$
P=\frac{\dot{\rho}_{1}}{\dot{\rho}} P_{1}+\frac{\dot{\rho}_{2}}{\dot{\rho}} P_{2}
$$

While the individual quantities $\dot{p}_{A} / \dot{\rho}_{A}$ are duality invariant,

$$
\frac{\dot{p_{1}}}{\dot{\bar{\rho}}_{1}}=\frac{\dot{p}_{1}}{\dot{\rho}_{1}}=\frac{1}{3}, \quad \frac{\dot{p_{2}}}{\dot{\bar{\rho}_{2}}}=\frac{\dot{p}_{2}}{\dot{\rho}_{2}}=-\frac{7}{3},
$$


the total adiabatic sound speed is not. It transforms as

$$
\frac{\dot{\bar{p}}}{\dot{\bar{\rho}}}=-\frac{\dot{p}}{\dot{\rho}}-2
$$

Again, an equation of state $p=-\rho$ is the only case of an invariant "sound speed". Combining (5.6), (5.11) and (5.15) it follows that the notion of adiabatic perturbations is duality invariant:

$$
\bar{P}=\frac{\dot{\bar{p}}}{\dot{\bar{\rho}}} \bar{D} \quad \Leftrightarrow \quad P=\frac{\dot{p}}{\dot{\rho}} D
$$

Non-adiabatic pressure perturbations

$$
P-\frac{\dot{p}}{\dot{\rho}} D=-3 H \frac{\dot{p}}{\dot{\rho}}\left[\frac{\hat{p}}{\dot{p}}-\frac{\hat{\rho}}{\dot{\rho}}\right] \neq 0
$$

are generally characterized by

$$
\begin{aligned}
P-\frac{\dot{p}}{\dot{\rho}} D= & \frac{\dot{\rho}_{1}}{\dot{\rho}}\left(P_{1}-\frac{\dot{p}_{1}}{\dot{\rho}_{1}} D_{1}\right)+\frac{\dot{\rho}_{2}}{\dot{\rho}}\left(P_{2}-\frac{\dot{p}_{2}}{\dot{\rho}_{2}} D_{2}\right) \\
& +\frac{\dot{\rho}_{1} \dot{\rho}_{2}}{\dot{\rho}^{2}}\left[\frac{\dot{p}_{2}}{\dot{\rho}_{2}}-\frac{\dot{p}_{1}}{\dot{\rho}_{1}}\right]\left[D_{2}-D_{1}\right]
\end{aligned}
$$

The first two terms on the right-hand side describe internal non-adiabatic perturbations within the individual components. The last term takes into account non-adiabatic perturbations due to the two-component nature of the medium. It is straightforward to check that also the non-adiabatic pressure perturbations are duality invariant,

$$
\bar{P}-\frac{\dot{\bar{p}}}{\dot{\bar{\rho}}} \bar{D}=P-\frac{\dot{p}}{\dot{\rho}} D
$$

Since in our case both components are adiabatic on their own (recall that $\hat{p}_{1}=\hat{\rho}_{1} / 3$ and $\hat{p}_{2}=-7 \hat{\rho}_{2} / 3$ ), the first two terms on the right-hand side of (5.18) vanish identically.

\section{B. Metric perturbations}

A homogenous and isotropic background universe with scalar metric perturbations and vanishing anisotropic pressure can be characterized by the line element (longitudinal gauge, cf. [31])

$$
\mathrm{d} s^{2}=-(1+2 \psi) \mathrm{d} t^{2}+a^{2}(1-2 \psi) \delta_{\alpha \beta} \mathrm{d} x^{\alpha} \mathrm{d} x^{\beta} .
$$

The perturbation dynamics is most conveniently described in terms of the gauge-invariant variable 32 .

$$
\zeta \equiv-\psi+\frac{1}{3} \frac{\hat{\rho}}{\rho+p}=-\psi-H \frac{\hat{\rho}}{\dot{\rho}} .
$$

Corresponding quantities for the components are

$$
\zeta_{A} \equiv-\psi-H \frac{\hat{\rho}_{A}}{\dot{\rho}_{A}} .
$$

On large perturbation scales the variable $\zeta$ obeys the equation (cf [33, 34, 35])

$$
\dot{\zeta}=-H\left(P-\frac{\dot{p}}{\dot{\rho}} D\right) .
$$

The definition (5.21) is motivated by the circumstance that under infinitesimal coordinate transformations

$$
x^{n^{\prime}}=x^{n}-\xi^{n}(x)
$$

the quantities $\psi$ and $\rho$ behave as

$$
\psi^{\prime}=\psi-H \xi^{0}, \quad \hat{\rho}^{\prime}=\hat{\rho}+\dot{\rho} \xi^{0} .
$$

Obviously, it makes sense to define dual metric perturbations by

$$
\bar{\psi} \equiv-\psi \quad \Rightarrow \quad \bar{\zeta}=-\zeta
$$

where

$$
\bar{\zeta} \equiv-\bar{\psi}-\bar{H} \frac{\hat{\bar{\rho}}}{\dot{\bar{\rho}}}
$$

For dual adiabatic perturbations the quantity $\bar{\zeta}$ is approximately conserved in the same sense in which the variable $\zeta$ is conserved for usual adiabatic perturbations. For the components we have

$$
\bar{\zeta}_{1}=-\zeta_{2}, \quad \bar{\zeta}_{2}=-\zeta_{1}
$$

with the consequence that

$$
\bar{\zeta}_{2}-\bar{\zeta}_{1}=\zeta_{2}-\zeta_{1}
$$

Under these circumstances Eq. (5.23) takes the form

$$
\dot{\zeta}=-3 H \frac{\dot{\rho}_{1} \dot{\rho}_{2}}{\dot{\rho}^{2}}\left[\frac{\dot{p}_{2}}{\dot{\rho}_{2}}-\frac{\dot{p}_{1}}{\dot{\rho}_{1}}\right]\left[\zeta_{2}-\zeta_{1}\right],
$$

where

$$
\frac{\dot{p}_{2}}{\dot{\rho}_{2}}-\frac{\dot{p}_{1}}{\dot{\rho}_{1}}=-\frac{8}{3} \text {. }
$$

The simplest way to write an equation for $S \equiv \zeta_{2}-\zeta_{1}$ is in terms of the effective interaction pressure $\Pi \equiv-\Gamma \rho_{2} /(3 H)$ (cf. Eqs. (3.19) and (3.20) $)$. Obviously, this quantity is invariant under duality transformations, i.e., $\bar{\Pi}=\Pi$. The equation is

$$
\dot{S}=3 H \dot{\Pi} \frac{\dot{\rho}}{\dot{\rho}_{1} \dot{\rho}_{2}}\left[\zeta_{\Pi}-\zeta+\frac{\dot{\rho}_{2}-\dot{\rho}_{1}}{\dot{\rho}} S\right] .
$$


The gauge invariant quantity $\zeta_{\Pi}$ describes the perturbation of the interaction term. It is defined in analogy to the other perturbation quantities,

$$
\zeta_{\Pi} \equiv-\psi-H \frac{\hat{\Pi}}{\dot{\Pi}}, \quad \Pi=-\frac{\Gamma \rho_{2}}{3 H} .
$$

From (2.11) we find the perturbed three curvature

$$
\hat{\mathcal{R}}=2\left(-\frac{2}{3} \hat{\Theta} \Theta+\hat{\rho}\right) .
$$

Since on large perturbation scales (upon neglecting spatial gradient terms)

$$
\frac{\hat{\rho}}{\rho}=2 \frac{\hat{\Theta}}{\Theta}
$$

holds, we obtain with (2.10) the (duality invariant) result $\hat{\mathcal{R}}=0$, i.e., vanishing three curvature perturbations.

Generally, the behavior of perturbations under a transformation between contracting and expanding phases will be of interest if one wants to trace back primeval postbig bang perturbations that are responsible for structure formation in our Universe to inhomogeneities in a contracting pre-big bang phase $1,1,2,3,4,4,5,6,7,8,29,10]$. While we do not aim to study specific scenarios of such a type in this paper, we shall briefly characterize a simple toy model for non-adiabatic perturbations and see whether and how it exhibits the expected behavior.

To this purpose we consider the special case $\dot{\epsilon}=0$ but we allow for spatial variations $\hat{\epsilon} \neq 0$ (cf. [30]). This is a simple way to equip the medium with an internal structure. A fluctuation of $\hat{\epsilon} \neq 0$ corresponds to a fluctuation of the equation of state parameter $p / \rho$,

$$
\left(\frac{p}{\rho}\right)^{\wedge}=-\frac{8}{3} \frac{\hat{\epsilon}}{(1+\epsilon)^{2}}
$$

Alternatively, using the interaction rate $\Gamma$ in (3.24), we may write

$$
\left(\frac{p}{\rho}\right)^{\wedge}=\frac{1}{3}\left(\frac{\Gamma}{H}\right)^{\wedge}
$$

which relates the fluctuations of the equation of state parameter to fluctuations of the ratio between the interaction rate and the Hubble rate. Fluctuating interaction rates are known to produce curvature perturbations in certain inflationary scenarios [36, 37, 38]. Here, in a different context, they are used to characterize an internal structure within the cosmic substratum [30, 39].
Under the given conditions we have $\hat{\rho}_{2}=\epsilon \hat{\rho}_{1}+\hat{\epsilon} \rho_{1}$ and the quantity $\zeta_{2}-\zeta_{1}$ on the right hand side of Eq. [5.30) becomes

$$
\zeta_{2}-\zeta_{1}=S=\frac{1}{3 \gamma} \frac{\hat{\epsilon}}{\epsilon}
$$

Given this expression, Eq. 5.32) then fixes the time dependence of $\hat{\epsilon}$.

With (5.38) equation (5.30) for $\zeta$ simplifies to

$$
\dot{\zeta}=2 H \frac{\hat{\epsilon}}{1-\epsilon^{2}},
$$

or, in terms of $(\Gamma / H)$,

$$
\dot{\zeta}=-\frac{H}{3 \gamma}\left(\frac{\Gamma}{H}\right)^{\wedge}
$$

This demonstrates that $\hat{\epsilon} \neq 0$, equivalent to $(\Gamma / H) \neq 0$, is necessary to have a non-adiabatic contribution at all. Only a fluctuating $\epsilon$ can give rise to non-adiabatic perturbations. Adiabatic perturbations are characterized by $(\Gamma / H)=0$ and $\zeta=$ const. For $(\Gamma / H)=0$ the two-component picture does not add any new feature to the perturbation dynamics of the single component description. With $\hat{\bar{\epsilon}}=\overline{\hat{\epsilon}}$ the transformation property of the perturbed ratio $\Gamma / H$ is

$$
\overline{\left(\frac{\Gamma}{H}\right)}=-\left(\frac{\Gamma}{H}\right)
$$

The equation (5.40) preserves its form for $H \rightarrow-H, 1+$ $p / \rho \rightarrow-1-p / \rho,(\Gamma / H) \rightarrow-(\Gamma / H) \hat{)} \zeta \rightarrow-\zeta$. Obviously, the case $\gamma=1+p / \rho=0$, equivalent to $\epsilon=1$, is singular and requires $\hat{\epsilon}$ to vanish. Of course, a toy model with $\dot{\epsilon}=0$ cannot describe a dynamical evolution to $\epsilon=1$. Nevertheless we believe this simple model to be useful to demonstrate some general features of the perturbation dynamics under duality transformations.

With (5.33), where $\Pi=\left(\gamma_{2}-\gamma_{1}\right) \rho_{2} /(1+\epsilon)$, and (5.38) we obtain

$$
\zeta_{\Pi}-\zeta=\frac{1-\epsilon}{1+\epsilon} S
$$

for the first term in the bracket on the right hand side of Eq. 5.32). Since the second term is

$$
\frac{\dot{\rho}_{2}-\dot{\rho}_{1}}{\dot{\rho}} S=\frac{\epsilon-1}{\epsilon+1} S,
$$


it follows that

$$
\dot{S}=0 \quad \Rightarrow \quad S=\text { const }
$$

i.e., our toy model is characterized by a constant value of the isocurvature quantity $S$. A constant $S$ implies that $\hat{\epsilon}$ and $(\Gamma / H)$ are constant as well. Notice that $\bar{S}=S$ (cf. Eqs. (5.29) ) is consistent with Eqs. (5.38) and (5.41).

For a constant $(\Gamma / H)$ equation (5.40) may be integrated to yield

$$
\zeta=\zeta_{i}-\frac{1}{3 \gamma}\left(\frac{\Gamma}{H}\right)^{\wedge} \ln \frac{a}{a_{i}}
$$

where the subscript $i$ denotes some initial value. A fluctuating interaction rate causes a variation in the curvature perturbation which is logarithmic in the scale factor.

\section{DISCUSSION}

Spatially flat cosmological models of General Relativity are invariant with respect to a duality transformation that converts expanding into contracting scenarios and vice versa. We have shown that this property singles out a duality invariant decomposition of any cosmic medium into two components with fixed equations of state. One of them is radiation, the other one is a phantom fluid, called dual radiation, characterized by a barotropic index $-4 / 3$. The specific features of the cosmic medium with a generally time depend total equation of state are encoded in the interaction between radiation and dual radiation. The appearance of internal interactions within the cosmic medium as the result of a symmetry requirement may be seen as an analog to corresponding properties of gauge theories. On the basis of the split into radiation and dual radiation we derived the behavior of a system of matter and radiation and of a mixture of cold dark matter and dark energy under duality transformations. In general, the duality transformation of a non-interacting mixture of two cosmic fluids will result in an interacting two-component system. Further examples of this decomposition are a fluid with constant equation of state, minimally and conformally coupled scalar fields and an extended Chaplygin gas. Both scalar field configurations belong to the subclass for which one of the components has constant energy density. The enlarged Chaplygin gas represents an example for a non-linear dependence of the component energy densities on the total energy density. The only duality invariant equation of state is $p=-\rho$, i.e., a de Sitter universe, dual to its anti de Sitter counterpart. For this case the energy densities of radiation and dual radiation are the same, i.e., each of them contributes a fraction of one half to the total energy density.

Finally, we provided an outline for the behavior of cosmological perturbations under duality transformations. In particular, we demonstrated that the concept of adiabatic perturbations is duality invariant. For a simple toy model we also calculated a non-adiabatic contribution to the curvature perturbation which is characterized by a logarithmic dependence on the scale factor.

\section{Acknowledgments}

This work was partially supported by the University of Buenos Aires under Project X224 and Consejo Nacional de Investigaciones Científicas y Técnicas under Project 5169 (LPC). W.Z. acknowledges support by the Brazilian grants 308837/2005-3 (CNPq) and 093/2007 (CNPq and FAPES).
[1] D. Wands, Class. Quantum Grav.19, 3403 (2002).

[2] F. Finelli and R. Brandenberger, Phys. Rev. D 65, 103522 (2002).

[3] R. Durrer and F. Vernizzi, Phys. Rev. D 66, 083503 (2002).

[4] Ch. Gordon and N. Turok, Phys. Rev. D 67, 123508 (2003).

[5] M. Gasperini and G. Veneziano, Phys. Rept. 373, 1 (2003).

[6] J. Martin and P. Peter, Phys. Rev. D 68, 103517 (2003).

[7] L.A. Boyle, P.J. Steinhardt, and N. Turok, hep-th/0403026

[8] Y.S. Piao and Y.Z. Zhang, Phys. Rev. D 70, 043516 (2004).

[9] Y.S. Piao, Phys. Lett. B 606, 245 (2005).
[10] J.A. Lidsay, gr-qc/0405055

[11] G. Veneziano, Phys. Lett. B265, 287 (1991).

[12] A.A. Tseytlin, Mod. Phys. Lett. A 6, 1721 (1991).

[13] A. Sen, Phys. Lett. B271, 295 (1991).

[14] M. Gasperini, hep-th/9907067.

[15] E.W. Kolb and M.S. Turner, The Early Universe, Addison Wesley, 1998.

[16] W. Zimdahl and D. Pavón, Mon. Not. R. Astron. Soc. 266, 872 (1994).

[17] L.P. Chimento and D. Pavón, Phys. Rev. D 73, 063511, (2006).

[18] C. Wetterich, Nucl. Phys. B 302, 668 (1988); ibid., Astron. Astrophys. 301, 321 (1995).

[19] L. Amendola, Phys. Rev. D 62, 043511 (2000); W. Zimdahl, D. Pavón and L.P. Chimento, Phys. Lett. 
B 521, 133 (2001); L.P. Chimento, A.S. Jakubi, D. Pavón, and W. Zimdahl, Phys. Rev. D 67, 083513 (2003); L. Amendola, Phys. Rev. D 69, 103524 (2004); Rong-Gen Cai and Anzhong Wang JCAP 03(2005)002; Zong-Kuan Guo, Rong-Gen Cai and Yan-Zhong Zhang, JCAP 05(2005)002; M.S. Berger and H. Shojaei, astro-ph/0606408

[20] Yifu Cai, Hong Li, Yun-Song Piao and Xinmin Zhang, gr-qc/0609039

[21] Jun-Qing Xia, Gong-Bo Zhao, Bo Feng, Hong Li, and Xinmin Zhang, Phys. Rev. D 73, 063521, (2006).

[22] S. Nesseris and L. Perivolaropoulos, astro-ph/0610092.

[23] V. Faraoni, Phys. Rev. D 68, 063508 (2003); E. Gunzig, A. Saa, L. Brenig, V. Faraoni, T. M. Rocha Filho and A. Figueiredo, Phys. Rev. D 63, 067301 (2001); V. Faraoni, Phys. Rev. D 53, 6813, (1996).

[24] P. F. Gonzalez-Diaz, Phys. Rev. D 68 (2003) 021303.

[25] L. P. Chimento and R. Lazkoz, Class.Quant.Grav. 23, (2006) 3195-3204.

[26] M. C. Bento, O. Bertolami and A. A. Sen, Phys. Rev. D 66, 043507 (2002).

[27] L.P. Chimento, Phys. Rev. D 69 (2004) 123517.

[28] L.P. Chimento, A.S. Jakubi, D. Pavón, and W. Zimdahl,
Phys. Rev. D 67, 083513 (2003).

[29] M.C. Bento, O. Bertolami, and A.A. Sen, Phys. Rev. D 70, 083519 (2004).

[30] W. Zimdahl and J.C. Fabris, Class. Quantum Grav.22, 4311 (2005).

[31] A.R. Liddle and D.H. Lyth, Cosmological Inflation and Large-Scale Structure Cambridge University Press, Cambridge 2000 .

[32] J.M. Bardeen, P.J. Steinhardt, and M.S. Turner, Phys. Rev. D 28, 679 (1983).

[33] D.H. Lyth, Phys. Rev. D 31, 1792 (1985).

[34] J. García-Bellido and D. Wands, Phys. Rev. D 53, 5437 (1996)

[35] D. Wands, K.A. Malik, D.H. Lyth, and A.R. Liddle, Phys. Rev. D 62, 043527 (2000).

[36] G. Dvali, A. Gruzinov, and M. Zaldarriaga, Phys. Rev. D 69, 083505 (2004).

[37] S. Matarrese and A. Riotto, JCAP 0308, 007 (2003).

[38] D.H. Lyth, C. Ungarelli, and D. Wands, Phys. Rev. D 67, 023503 (2003).

[39] W. Zimdahl, Int. J. Mod. Phys. D 14, 2319 (2005). 